\title{
Monolithic Stationary Phases for Capillary Electrochromatography Based on Synthetic Polymers: Designs and Applications
}

\author{
Frantisek Svec, Eric C. Peters ${ }^{1)}$, David Sýkora, Cong Yu, Jean M. J. Fréchet* \\ Department of Chemistry, University of California, Berkeley, CA 94720-1460, USA
}

Ms received: September 29, 1999; accepted: November 3, 1999

Key Words: Capillary electrochromatography; monolithic columns; synthetic polymers; stationary phase

\begin{abstract}
Summary
Monolithic materials have quickly become a well-established stationary phase format in the field of capillary electrochromatography (CEC). Both the simplicity of their in situ preparation method and the large variety of readily available chemistries make the monolithic separation media an attractive alternative to capillary columns packed with particulate materials. This review summarizes the contributions of numerous groups working in this rapidly growing area, with a focus on monolithic capillary columns prepared from synthetic polymers. Various approaches employed for the preparation of the monoliths are detailed, and where available, the material properties of the resulting monolithic capillary columns are shown. Their chromatographic performance is demonstrated by numerous separations of different analyte mixtures in variety of modes. Although detailed studies of the effect of polymer properties on the analytical performance of monolithic capillaries remain scarce at this early stage of their development, this review also discusses some important relationships such as the effect of pore size on the separation performance in more detail.
\end{abstract}

\section{Introduction}

In contrast to mechanical pumping that relies on moving parts, electroendoosmotic flow (EOF) is generated within a stationary system by applying an electrostatic potential across the entire length of a device, such as a capillary or a flat profile cell. The potential advantages of the flat flow profile generated by EOF in both thin-layer and column chromatography were recognized as early as in 1974 by Pretorius [1]. Although the first electrochromatographic separations in packed capillary columns (CEC) were demonstrated in the early 1980s [2-4], serious technical difficulties have slowed the further development of this promising separation method $[5,6]$. Since the revival of interest in CEC in the mid 1990's resulting from the search for new miniaturized separation methods with vastly enhanced efficiencies and peak capacities, research activity in this field has rapidly expanded, and the number of published papers has grown exponentially.

CEC is often presented as a hybrid method that combines the capillary column format and electroosmotic flow employed in high-performance capillary electrophoresis (HPCE) with

1) Present address: Novartis Institute of Functional Genomics, San Diego, CA 92121-1125, USA. the use of a solid stationary phase and a separation mechanism characteristic of HPLC based on specific interactions of solutes with a stationary phase. Therefore CEC is most commonly implemented by means typical of both HPLC (packed columns) and HPCE (use of electrophoretic instrumentation). To date, both columns and instrumentation developed specifically for CEC remain scarce.

Although numerous groups around the world prepare CEC columns using a variety of approaches, the vast majority of these efforts mimic in one way or another standard HPLC column technology. However, aspects of this technology have proven difficult to implement on the capillary scale. Additionally, the stationary phases packed in CEC capillaries are often standard commercial HPLC-grade beads. Since these media are tailored for regular HPLC modes, and their surface chemistries are optimized accordingly, their use incorrectly treats CEC as a subset of HPLC. Truly optimized, CEC packings should play a dual role: in addition to providing sites for the required interactions (as in HPLC), they must also be involved in electroosmotic flow. As a result, packings that are excellent for HPLC may offer limited performance in the CEC mode. This realization of the basic differences between HPLC and CEC [7] has stimulated the development of both specific particulate packings having properties tuned to the needs of CEC as well as alternative column technologies.

Numerous papers have already demonstrated the successful use of packed capillary columns in CEC-based separations. The preparation of these columns includes two key steps: the fabrication of retaining frits within a capillary as well as the subsequent packing of small diameter particles into narrowbore tubes. Both of these steps require considerable experimental skill and experience in order to obtain stable columns with reproducible properties.

\section{Monolithic Capillary Columns}

The technical difficulties associated with packed columns have spurred the development of various alternative approaches. For example, one of these competing technolo- 
gies - in situ polymerized organic separation media - was adopted from a concept developed for much larger diameter HPLC columns. As a result of their unique properties, these monolithic materials have recently attracted considerable attention from a number of different research groups. Perhaps the most appealing aspect of the monolithic materials is their ease of preparation, as shown schematically in Figure 1. The simple in situ polymerization process performed directly within the confines of a mold avoids the problems of both frit formation and packing. Additionally, columns of virtually any length are easily accessible. The polymerization mixture may also be prepared using a wide variety of monomers, allowing a nearly unlimited choice of both matrix and surface chemistries. This flexibility enables the easy tailoring of both the interactions that are required for specific separation modes as well as the level of EOF generated by the support. Finally, the control that can be exerted over the polymerization process enables the facile optimization of the porous properties of the monolith that, in turn, may directly affect the flow rate and chromatographic efficiency of the system.

\subsection{Acrylamide Based Porous Polymer Monoliths Prepared from Aqueous Solutions}

\subsubsection{Polymer Gels}

The first monolithic CEC columns contained swollen hydrophilic polyacrylamide gel, mimicking those used for capillary gel electrophoresis [8]. Typically, the capillary is filled with an aqueous polymerization mixture containing monovinyl and divinyl (crosslinking) acrylamide-based monomers as well as a free radical redox initiating system, such as ammonium peroxodisulfate and tetramethylethylenediamine (TEMED). Since initiation of the polymerization process begins immediately upon mixing of all the components at room temperature, the reaction mixture must be used immediately. Typically, the polymerization process is allowed to proceed overnight to afford capillaries filled with continuous gel beds. It should be noted that these gels are very loose, highly swollen materials that usually contain no more than $5 \%$ solid polymer.

For example, Fujimoto [9] polymerized an aqueous solution of acrylamide, methylenebisacrylamide, and 2-acrylamido-2methyl-1-propanesulfonic acid (AMPS) within the confines of a capillary. Despite the lack of chemical attachment to the inner wall of the capillary, these crosslinked gels showed fair physical stability. Although column efficiencies of up to 150000 plates/m were observed for acetophenone, retention times on these columns were prohibitively long. This behavior was probably due, in part, to the relatively high background buffer concentration of $0.1 \mathrm{~mol} / \mathrm{l}$ employed. This concentration is at least one order of magnitude higher than that typically used in current CEC studies. Based on his results, Fujimoto concluded that the prevailing mechanism of the

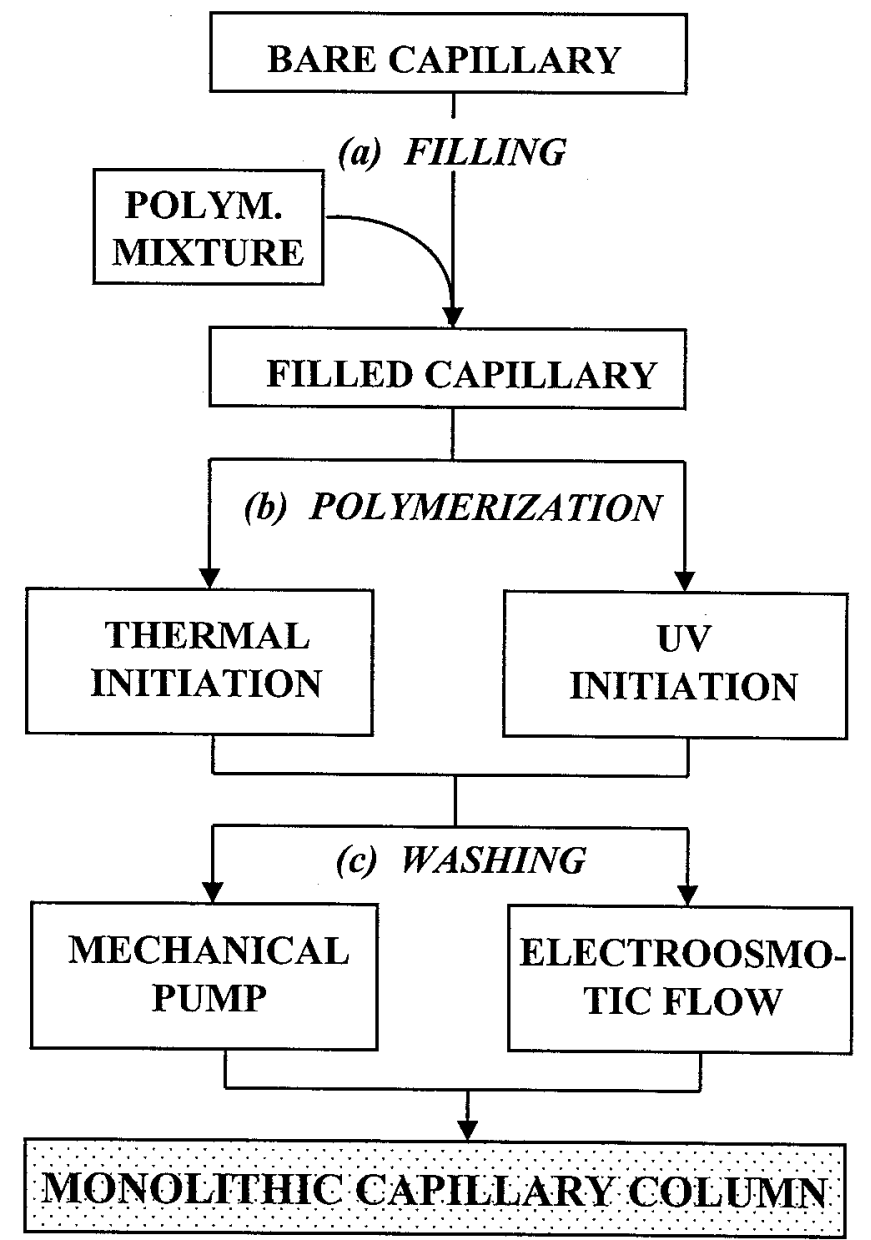

Figure 1. Schematics for the preparation of monolithic capillary columns. First, the bare capillary is filled with the polymerization mixture (step a) that contains functional monomer, crosslinking monomer, initiator, and porogenic solvent. Polymerization (step b) is then initiated thermally or by UV irradiation to afford a rigid monolithic porous polymer. The resulting monolith within the capillary is washed (step c) with the mobile phase using a pump or electroosmotic flow and used as a CEC column.

separation was sieving rather than an interaction of the solutes with the matrix [10].

Replacement of the hydrophilic acrylamide with the more hydrophobic $N$-isopropylacrylamide, in combination with the pre-functionalization of the capillary internal surface with 3(trimethoxysilyl)propyl methacrylate, afforded a monolithic gel covalently attached to the capillary. The electrochromatographic elution of hydrophobic analytes from this column required the use of aqueous buffer/acetonitrile mixtures [11]. In contrast to the previously described work, improvements in the separations were observed using these "fritless" hydrogel columns. This is confirmed by reports of both shorter retention times and column efficiencies as high as 160,000 for various steroids [11]. The separations of hydrophobic compounds obtained using this polymer gel stationary phase 
exhibit many of the attributes typical of reversed-phase chromatography, including a linear dependence of the retention factor $k^{\prime}$ on the composition of the mobile phase. This led to the conclusion that, in contrast to the original polyacrylamide-based gels, size-exclusion was no longer the primary mode of separation.

\subsubsection{Highly Crosslinked Monoliths}

Another approach towards continuous CEC beds involving highly crosslinked acrylamide polymers was reported by Hjertén [12] at about the same time [9, 10]. Hjertén's original approach was complex, requiring a multiplicity of steps including the modification of the capillary surface with 3-(trimethoxysilyl)propyl methacrylate, two individual polymerizations, and a chemical functionalization [13]. The initial polymer matrix was formed by copolymerizing a dilute aqueous solution of 2-hydroxyethyl methacrylate and piperazine diacrylate using a standard redox system in the presence of a high concentration of ammonium sulfate. The pores of this matrix were then filled with another polymerization mixture containing allyl glycidyl ether and dextran sulfate, and the second polymerization proceeded within the pores of the initial matrix leading to the "immobilization" of the charged dextran within the newly formed composite. Eventually, reaction of both epoxide and hydroxyl functionalities with 1,2epoxyoctadecane led to the covalent functionalization of the matrix with a number of $\mathrm{C}_{18}$ chains. Several chromatographic measurements were performed using these capillaries, with retention times in excess of 20 min being required for the elution of aromatic hydrocarbons [13].

In order to simplify the tedious preparation method, a simpler procedure was later developed by the same group [14]. The polymerization mixture consisted of an aqueous solution of acrylamide, piperazine diacrylamide, and vinylsulfonic acid with added stearyl methacrylate or butyl methacrylate to control the hydrophobicity of the gel. Since neither of these nonpolar monomers is soluble in water, a surfactant was added to the mixture, followed by sonication to form an emulsion of the hydrophobic monomer in the aqueous solution. Once initiated, the mixture was immediately drawn into an acryloylsilanized capillary, where the polymerization was completed. The presence of the strongly acidic sulfonic acid functionalities afforded EOF that remained constant over a broad $\mathrm{pH}$ range.

Although the initial separations performed using these continuous gel beds were good, an ingenious trick involving changes in the strength of the mobile phase also enabled further improvements in the resolution of various polycyclic aromatic hydrocarbons (PAH). Therefore, a solution containing the analytes dissolved in 50/50 acetonitrile/aqueous buffer was injected electrophoretically into the capillary column, and the separation was started. After a short period of time, the solvent in the inlet vial was replaced with a 70/30 acetoni-

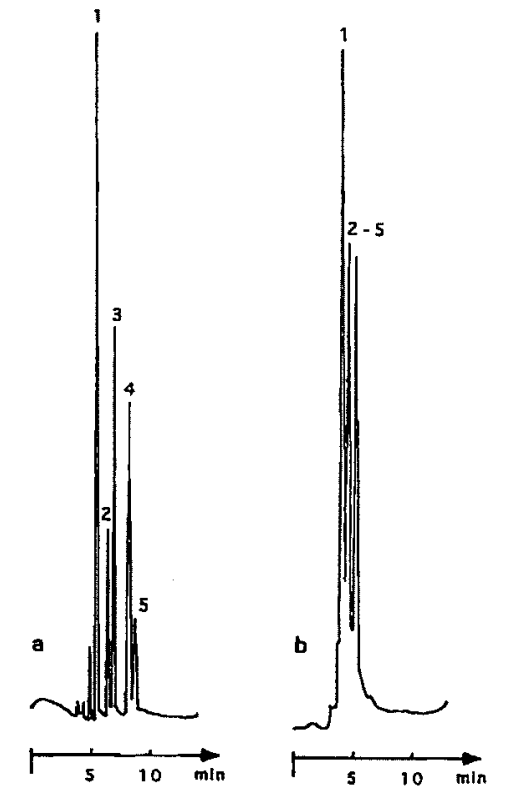

Figure 2. Effect of sodium dodecylsulfate in the mobile phase upon electrochromatography of polycyclic aromatic hydrocarbons on a C18derivatized continuous bed containing sulfonic acid groups (Reprinted with permission from ref. 14. Copyright 1996 American Chemical Society). Capillary: $14 \mathrm{~cm}(10 \mathrm{~cm}$ effective length $) \times 100 \mu \mathrm{m}$ i.d. Applied voltage: $3.0 \mathrm{kV}$ Eluent: $60 \%(v / v)$ acetonitrile in $4 \mathrm{mmol} / \mathrm{l}$ sodium phosphate (pH 7.4). (a) $1.0 \mathrm{mmol} / \mathrm{l} \mathrm{SDS}$ added to the eluent; (b) without addition of SDS (control). Peaks: naphthalene (1), 2-methylnaphthalene (2), fluorene (3), phenanthrene (4), anthracene (5).

trile/buffer mixture, and the elution was completed under these conditions, leading to peaks sharper than those obtained using standard isocratic elution. The authors assumed that this improvement resulted from the gradient of the mobile phase generated by diffusion across the strong solvent/weak solvent interface. Addition of sodium dodecyl sulfate at levels below the critical micelle concentration was also reported to improve isocratic CEC separations [14]. Figure 2 shows the remarkable difference between two separations of aromatic hydrocarbons in the presence and in the absence of sodium dodecylsulfate.

The same group very recently described another method of preparation of a monolithic capillary column that was used for CEC gradient separation of proteins [15]. The first step involved a polymerization initiated by ammonium persulfate/ TEMED system in a two-phase system consisting of two phases: aqueous, consisting of a solution of acrylamide and piperazine diacrylamide in a mixture of a buffer solution and dimethylformamide, and immiscible highly hydrophobic octadecyl methacrylate. Continuous sonication was applied for $40 \mathrm{~min}$ in order to emulsify the highly hydrophobic octadecyl methacrylate and form a dispersion of fine polymer particles. In the remaining steps of this preparation process, another portion of initiator was added to the system to restart the polymerization of two newly added monomers, dimethyldiallylammonium chloride and piperazine diacrylamide. The 


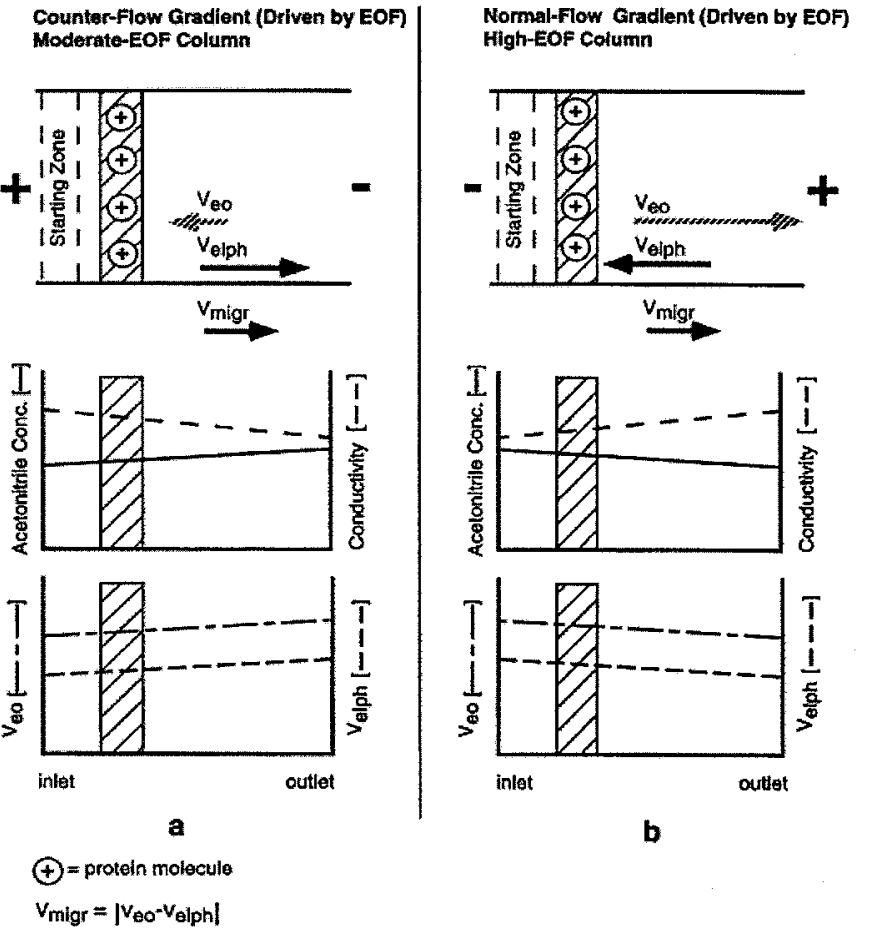

Figure 3. Diagram illustrating the principle for counterflow (a) and normal-flow (b) gradients (Reprinted with permission from ref. 15. Copyright 1999 American Chemical Society). The direction of electroosmotic flow is opposite to that of the electrophoretic movement in both methods and is opposite to the net migration velocity, $v_{\text {migr }}\left(=v_{\mathrm{eo}}-v_{\text {elph }}\right)$, in (a) and coincides with the net migration direction in (b). $v_{\text {eo }}$ is not constant along the capillary, and $v_{\text {elph }}$ is higher in the direction of the electrophoretic migration.

resulting partly polymerized dispersion was then forced into a methacryloylsilylated capillary using pressure and, finally, the polymerization process was re-started and carried out to completion.

Understanding and controlling the forces that drive the movement of charged molecules during CEC separations is extremely important. Proteins may be particularly difficult to separate, since, depending on their net charge as determined by both the protein $\mathrm{pI}$ and the $\mathrm{pH}$ value of the mobile phase, the same molecule can move electrophoretically towards either the anode or the cathode. In contrast, EOF proceeds in only one direction as determined by the charge of the mobile counterions in the Stern layer. Thus, in Hjertén's approach, using immobilized quaternary ammonium cations, EOF always proceeds from the anode to the cathode. Electroosmotic flow was also used by the same group to introduce the mobile phase gradient (generated using an HPLC instrument to mix the gradient) into the capillary. Two overall flow scenarios shown schematically in Figure 3 were discussed [15]:

(i) In the monolith containing a high level of charged moieties, electroosmotic flow outweighs the electrophoretic

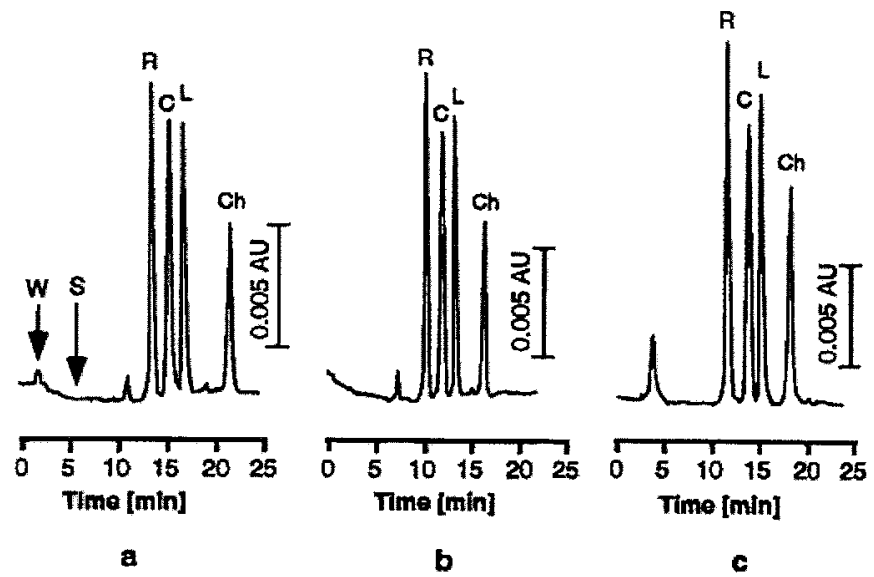

Figure 4. Separation of proteins using gradient electrochromatography (a, b) and reversed-phase $\mu$ HPLC (c) (Reprinted with permission from ref. 15. Copyright 1999 American Chemical Society). Peaks: ribonuclease A (R); cytochrome c (C); lysozyme (L); chymotrypsinogen $(\mathrm{Ch})$ Protein concentration: $0.6 \mathrm{mg} / \mathrm{mL}$ of each protein except for ribonuclease A $(1.8 \mathrm{mg} / \mathrm{mL})$; mobile phase: a linear gradient from 5 to $80 \%$ acetonitrile in $5 \mathrm{mmol} / \mathrm{L}$ sodium phosphate $\mathrm{pH} 2.0$; Detection at $280 \mathrm{~nm}$. Columns: $8 \mathrm{~cm}(6 \mathrm{~cm}$ effective length) $\times 50 \mu \mathrm{m}$ i.d.; voltage, $5.5 \mathrm{kV}(700$ $\mathrm{V} / \mathrm{cm}$ ); (a) Moderate-EOF column, (b) high-EOF column, (c) conventional capillary RP-HPLC; pressure $5 \mathrm{MPa}$.

migration. In this "normal flow gradient" situation, both the EOF and the net migration of the protein molecules occur in the same direction, provided that all of the proteins have net charges of equal sign. This was achieved by using a mobile phase consisting of $80 \%$ acetonitrile and $20 \%$ buffer with a $\mathrm{pH}$ value of 2 . This $\mathrm{pH}$ is well below the $\mathrm{pI}$ of the proteins, ensuring that the biopolymers were positively charged. Samples were injected at the cathode, and as with other typical gradient systems, the percentage of acetonitrile was higher at the capillary inlet (cathode) than at the outlet, with detection occurring at the anodic end of the column.

(ii) In contrast, in the monolith containing a moderate number of charged moieties, electromigration of the charged proteins was faster than the electroosmotic flow. In this "counter flow gradient" system, the EOF proceeded in the same direction. However, protein analytes were injected at the anode, and migrated in a direction opposite to the gradient due to their electrophoretic mobilities. Accordingly, the detector was positioned at the cathodic end of the capillary column.

Figure 4 shows excellent separations of four proteins in both moderate (a) and high EOF columns (b) using electrically driven flow, and compares the separation with that achieved using standard HPLC methodology (c). Since the separations were primarily governed by the nature of the mobile phase gradient, all three chromatograms are very similar. This comparison also demonstrates that the separation is achieved via reversed-phase partitioning rather than electrophoresis.

It should be noted that this successful approach to monolithic CEC columns for the separation of proteins employs a poly- 
meric matrix containing positively charged moieties. Since the overwhelming majority of proteins also possess net positive charges at $\mathrm{pH}=2$, the danger of undesired electrostatic interactions is greatly diminished. The occurrence of very strong electrostatic interactions most likely explains why, to date, all attempts to separate proteins on columns containing carboxylic or sulfonic acid moieties at $\mathrm{pH}$ values below the pI have failed [16].

\subsection{Acrylamide-Based Monolithic Columns Prepared in Organic Solvents}

Despite the undeniable success, the use of purely aqueousbased polymerization systems for the preparation of monolithic capillaries for CEC also has some limitations. Perhaps the greatest limitation is that the typical nonpolar monomers that are required to achieve the necessary hydrophobicity for a reversed-phase CEC bed are insoluble in water. In contrast to the "fixed" solubilizing properties of water, the wealth of organic solvents possessing polarities ranging from highly nonpolar to extremely polar enables the formulation of mixtures with solvating capabilities that may be tailored over a very broad range. An additional feature of organic solvents is their intrinsic ability to control the porous properties of the monoliths.

In contrast to the process of sonication used to disperse hydrophobic monomers in an aqueous buffer [14, 15], Novotny simplified the incorporation of highly hydrophobic ligands into acrylamide-based matrices [16] by using mixtures of aqueous buffer and $N$-methylformamide to prepare homogeneous polymerization solutions. The overall concentration of the monomers (acrylamide, methylene bisacrylamide, acrylic acid, and $\mathrm{C}_{4}, \mathrm{C}_{6}$, or $\mathrm{C}_{12}$ alkyl acrylate) in solution was kept constant throughout the study at the level of $5 \%$. The composition of the mixed buffer/methylformamide solvent depended on the type of alkyl methacrylate used, and ranged from $50 \% \mathrm{~N}$-methylformamide for butyl acrylate to 95\% for dodecyl acrylate. Columns with high efficiencies were only obtained when the polymerization was performed in the presence of poly(oxyethylene) $\left(M_{\mathrm{w}}=10000\right)$ dissolved in the polymerization mixture. Poly(oxyethylene) is known to induce lateral aggregation of acrylamide chains, thus contributing to the formation of more porous structures [17]. Polymerization was achieved using the usual peroxodisulfate/ TEMED initiating system within acryloylsilylated capillaries, affording monoliths possessing an opaque appearance characteristic of macroporous polymers. However, no analytical characterization of the pore structures was performed. Once the polymerization was complete, the poly(oxyethylene) and other low molecular weight compounds were washed out of the column using electroosmotic flow. This preparation method is remarkably reproducible.

All of the monoliths containing the various alkyl acrylates behaved as typical reversed-phase stationary phases, as evidenced by the linear decrease in their retention factors in

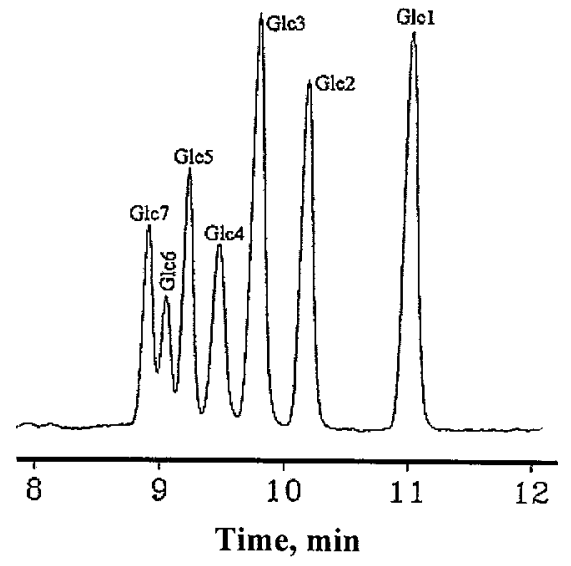

Figure 5. Isocratic electrochromatography of maltooligosaccharides (glucose (Glc1)-maltohexaose (Glc6)) in a capillary filled with a macroporous polyacrylamide/poly(ethylene glycol) matrix, derivatized with $\mathrm{C} 4$ ligand (15\%) and containing vinylsulfonic acid (10\%). 2-Aminobenzamide was used to "tag" the oligosaccharides for the laser-induced fluorescence detection. (Reprinted with permission from ref. 16. Copyright 1997 American Chemical Society). Conditions: capillary, $32 \mathrm{~cm}(25 \mathrm{~cm}$ effective length) $\times 100 \mu \mathrm{m}$ i.d.; mobile phase, $0.1 \%$ aqueous acetic acid containing $5 \%(\mathrm{v} / \mathrm{v})$ acetonitrile; field strength, $900 \mathrm{~V} / \mathrm{cm}, 20 \mathrm{~A}$; injection, $100 \mathrm{~V} / \mathrm{cm}$ for $5 \mathrm{~s}$; sample concentration, $5-10 \mu \mathrm{mol} / \mathrm{L}$.

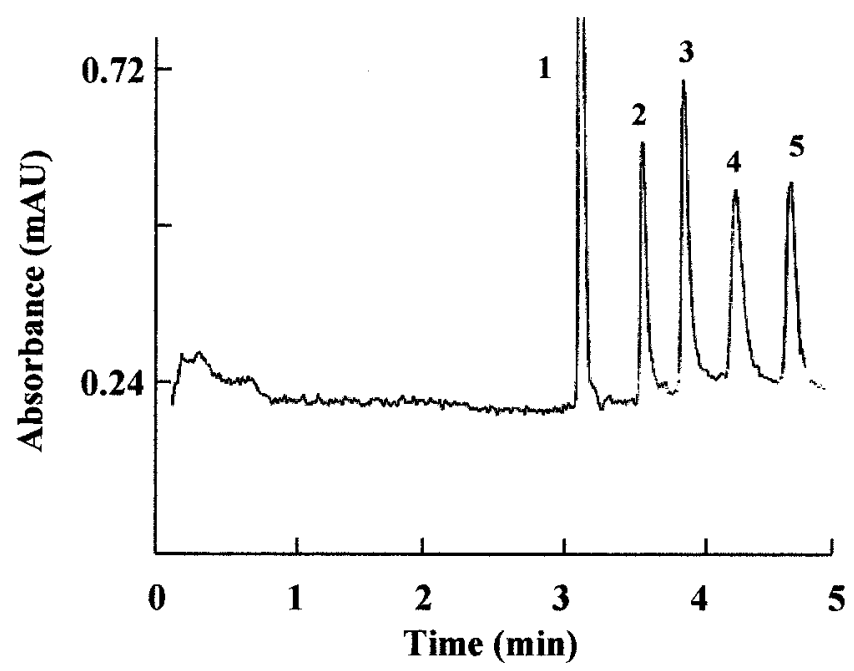

Figure 6. Isocratic electrochromatography of peptides in a capillary filled with a macroporous polyacrylamide/poly(ethylene glycol) monolith, derivatized with C12 ligands (29\%) and containing acrylic acid (Reprinted with permission from ref. 16. Copyright 1997 American Chemical Society). Conditions: mobile phase, $47 \%$ acetonitrile in a $10 \mathrm{mM}$ Tris/15 mM boric acid ( $\mathrm{pH} \mathrm{8.2)} \mathrm{buffer;} \mathrm{voltage,} 22.5 \mathrm{kV}$ (900 V/cm); sample concentration, $4-10 \mathrm{mg} / \mathrm{mL}$; UV detection at $270 \mathrm{~nm}$. Peaks: system peak (1), tyr-arg (2), gly-gly-tyr-arg (3), tyr-ala-gly-phe-leu-arg (D-ala ${ }^{3}$ leucine enkephalin-arg) (4), tyr-gly-gly-phe-leu- $\mathrm{NH}_{2}$ (leucine enkephalinamide) (5).

response to an increasing percentage of organic solvent in the mobile phase. Column efficiencies calculated for on-column detected peaks of phenylketones used as model analytes were in the range of 300000-400000 plates $/ \mathrm{m}$. These monolithic 


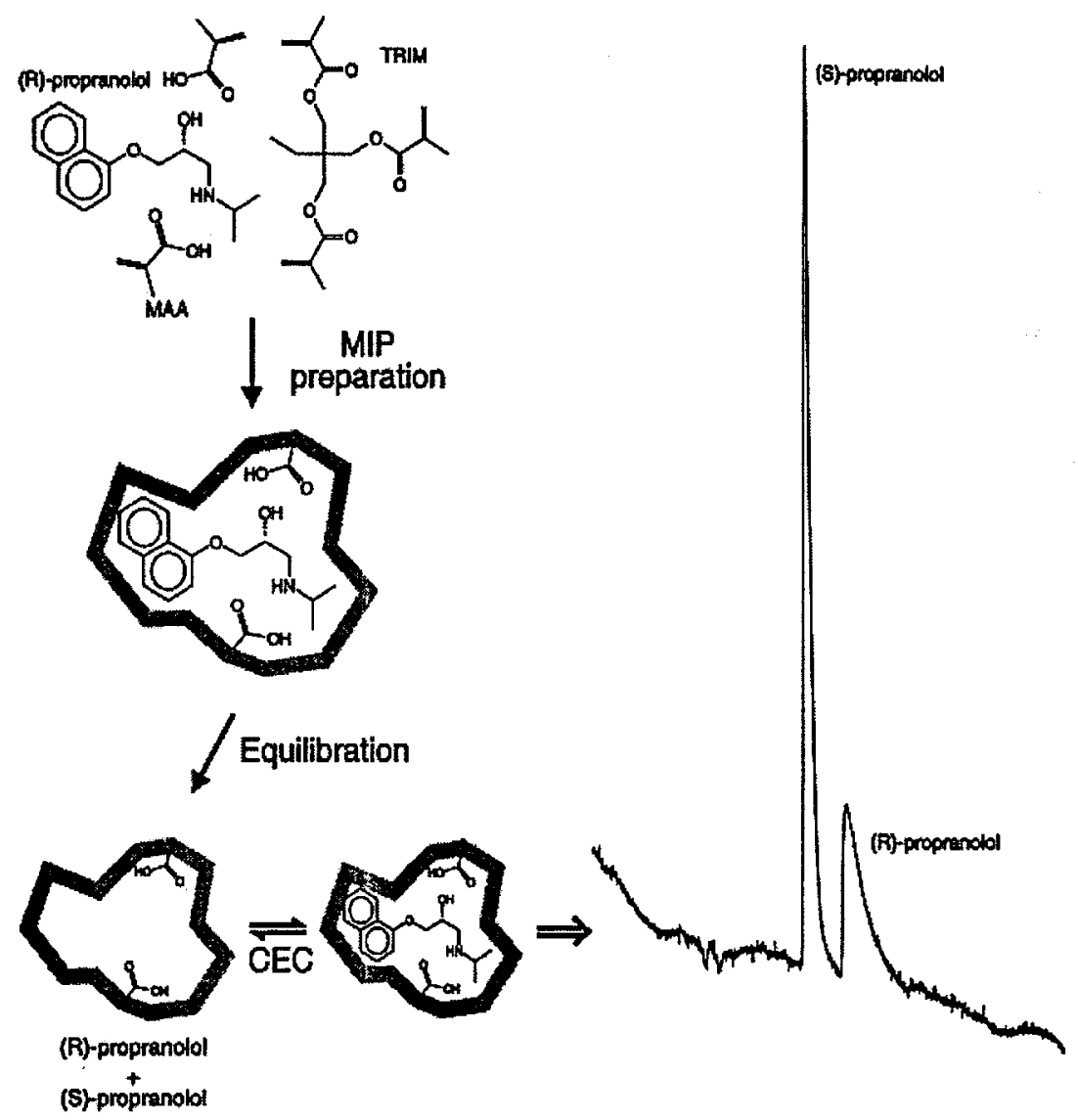

Figure 7. Molecular imprinting of (R)-propranolol using methacrylic acid (MAA) as the functional monomer and trimethylolpropane trimethacrylate (TRIM) as the crosslinking monomer. The enantioselectivity of a given polymer is predetermined by the configuration of the ligand, R-propranolol present during its preparation. Since the imprinted enantiomer possesses a higher affinity for the polymer, the separation is obtained with a predictable elution order of the enantiomers. (Reprinted with permission from ref. 26. Copyright 1998 Elsevier)

columns easily tolerated rather high loading levels without concomitant loss of efficiency, though excessive tailing of the peaks was observed under overload conditions.

In contrast to the typical hydrophobic aromatic model compounds often used, Novotny extended the range of potential analytes to include carbohydrates [16]. Since these compounds are best separated at low $\mathrm{pH}$ values, vinylsulfonic acid was incorporated into the monolith rather than acrylic acid in order to provide moieties that would support EOF under these conditions. Figure 5 shows a typical separation. Since oligosaccharides do not adsorb in the UV range, aminobenzamide tags were attached to the analyte molecules prior to the separation, and laser-induced fluorescence was used for their detection. Column efficiencies for glucose, maltose, and maltotriose were all found to be in the range of 190000230000 plates $/ \mathrm{m}$.

A monolithic CEC column incorporating dodecyl acrylate was also successfully used for the isocratic separation of charged molecules - oligopeptides (di-, tri, penta-, and hexa- peptide). Figure 6 shows the baseline separation that was achieved in isocratic mode in less than $5 \mathrm{~min}$ at $900 \mathrm{~V} / \mathrm{cm}$. The elution pattern and the efficiency of the separation were found to strongly depend on both the percentage of acetonitrile and the $\mathrm{pH}$ of the mobile phase, suggesting that a gradient elution method would have been even more appropriate. However, larger proteins could not be eluted isocratically.

\section{Imprinted Monolithic Columns for Chiral CEC}

Molecular imprinting has recently attracted considerable attention as an approach to the preparation of polymers containing recognition sites with predetermined selectivity. The history and specifics of the imprinting technique pioneered by Wulff in the 1970s have been detailed in several excellent review articles [18-20]. These materials, if successfully prepared, are expected to find applications in such areas as the resolution of racemates, substrate selective catalysis, and the production of "artificial antibodies". Imprinted monoliths 

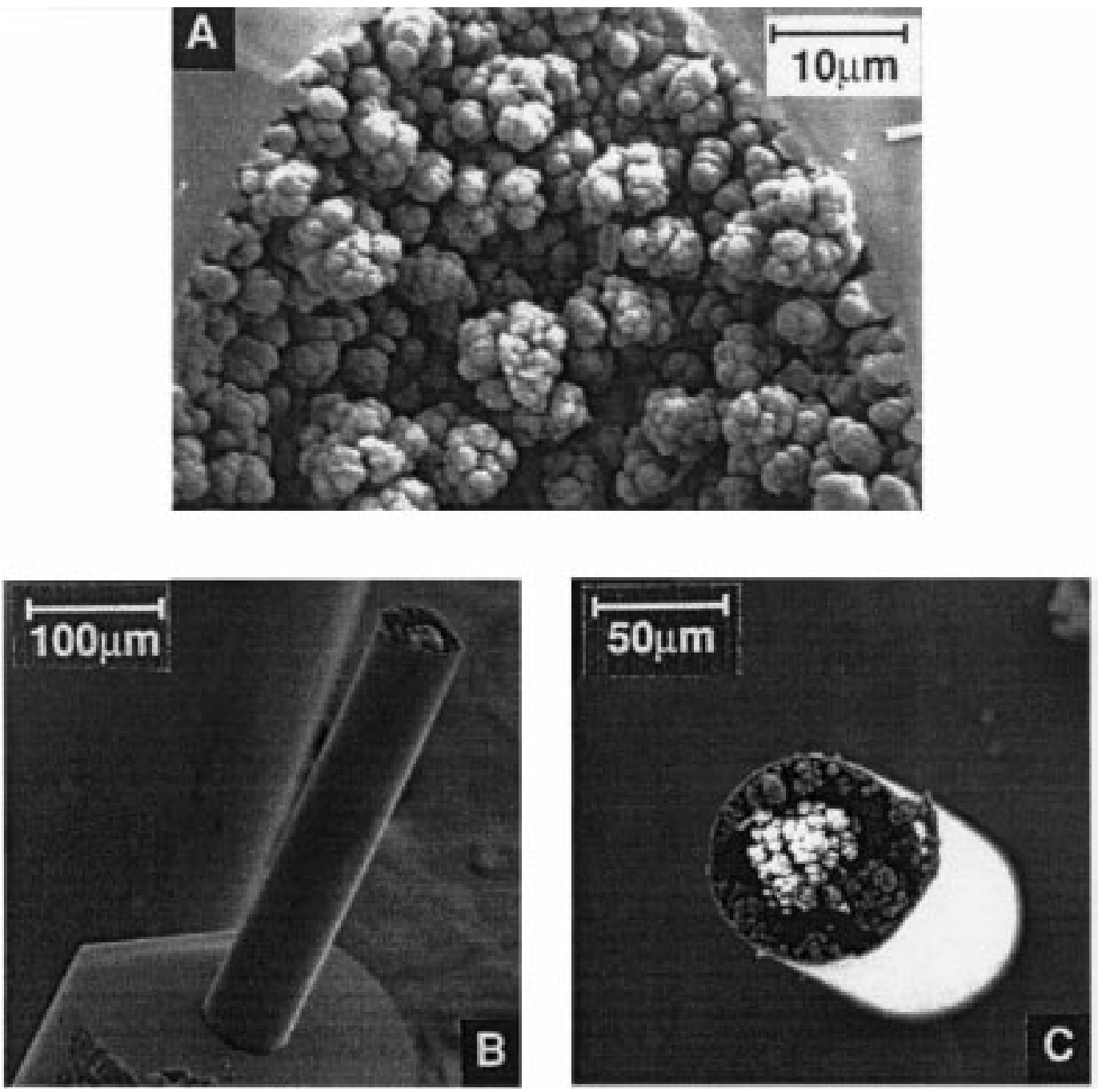

Figure 8. SEM micrographs of styrene-based monolithic capillary. (Reprinted with permission from ref. 28. Copyright 1999 Elsevier)

have also received attention recently as stationary phases for capillary electrochromatography.

The imprinting process shown schematically in Figure 7 involves the preorganization of functional monomer molecules such as methacrylic acid and vinylpyridine around a template molecule and subsequent copolymerization of this complex with a large amount of a crosslinking monomer (ethylene dimethacrylate-EDMA, trimethylolpropane trimethacrylate-TRIM) [21]. Under ideal conditions, imprints possessing both a defined shape and a specific arrangement of chemically interactive functional groups that reflect those of the templated molecule remain in the polymer after extraction of the template.

In the early pioneering days of this technique, the final imprinted materials possessed pores too small to support flow through the medium. Therefore, the material could only be used as crushed and sieved irregular particles. Truly monolithic technology was directly employed for CEC application only after the introduction of "superporous" imprinted monolithic capillaries by Nillson in 1997 [21-23]. Isooctane was used as a porogen in order to produce a macroporous structure with large pores without interfering with the imprinting process. The imprinted polymers were polymerized within capillaries having a vinylized inner surface using both thermally and UV light initiated polymerization [21-23]. Such "superporous" imprinted monoliths were successfully used for the separation of the enantiomers of propanolol (Figure 7), metoprolol, and ropivacaine. Using a similar process employing mixtures of ethylene dimethacrylate with methacrylic acid and/or 2-vinylpyridine, Lin developed imprinted monolithic columns for the CEC separation of racemic phenylalanine [24]. 


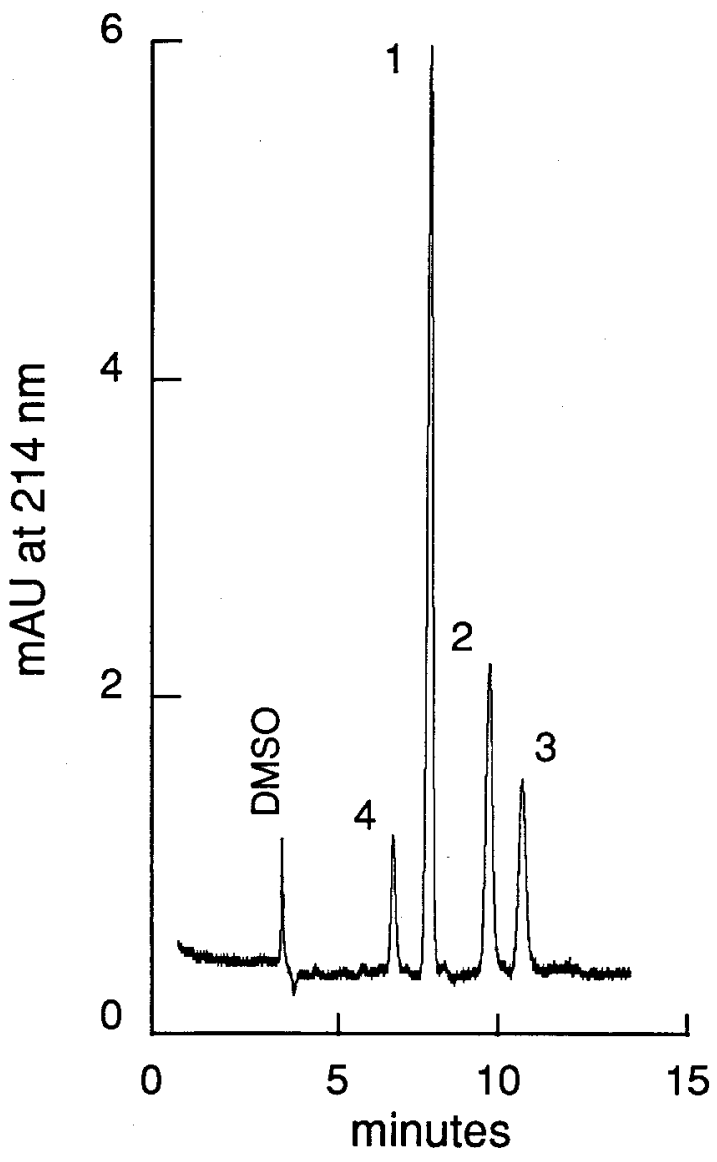

Figure 9. Electrochromatogram of acidic and basic peptides (Reprinted with permission from ref. 28. Copyright 1999 Elsevier). Column $31 \mathrm{~cm}$ (21 active length) $\times 75 \mu \mathrm{m}$, porous styrenic monolith with dimethyloctylammonium functionalities; mobile phase $25 \%$ acetonitrile in $5 \mathrm{mmol} / \mathrm{l}$ phosphate buffer $\mathrm{pH} 3.0$ containing $50 \mathrm{mmol} / \mathrm{l}$ sodium chloride; reversed polarity, electrokinetic injection for $2 \mathrm{~s}, 5 \mathrm{kV}$. Peaks: angiotensin II (1), angiotensin I (2), [ $\left.\mathrm{Sar}^{1}, \mathrm{Ala}^{8}\right]$-angiotensin II (3), insulin (4).

Lin's group also investigated a composite approach toward imprinted monoliths. A non-porous polymer imprinted with L-phenylalanine anilide was first prepared via UV initiated polymerization within a glass ampoule. This bulk polymer was ground into small irregular-shaped particles, sieved, and suspended in a solution of acrylamide and methylenebisacrylamide containing a redox initiator. The heterogeneous dispersion was then drawn into the capillary, and the polymerization was completed affording a gel-type monolith with immobilized solid particles. The capillary column was then used for the separation of D,L-phenylalanine [25]. Such approaches to monolithic imprinted stationary phases for CEC have been recently reviewed in greater detail [26].

Several attempts to produce chiral CEC monoliths have also been described. For example, Koide and Ueno prepared a monolithic gel for chiral resolution by polymerizing acrylamide, bisacrylamide, and AMPS in the presence of polymeric carboxymethyl- $\beta$-cyclodextrin [27]. This capillary col-
Scheme 1:

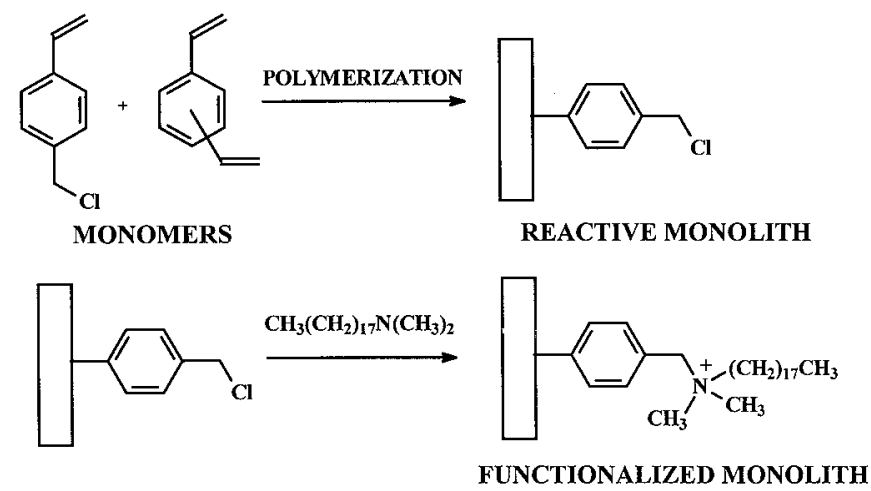

umn exhibited 26000 plates for the separation of terbutaline enantiomers, effecting baseline separation even with a separation factor $\alpha$ of only 1.03 .

\section{Polystyrene-Based Monolithic Capillary Columns}

Horváth's group has recently reported the preparation of porous rigid monolithic capillary columns for CEC by polymerizing mixtures of chloromethylstyrene, divinylbenzene, and azobisisobutyronitrile in the presence of various porogenic solvents such as methanol, ethanol, propanol, toluene, and formamide [28]. The capillary wall was silanized using a $50 \%$ dimethylformamide solution of 3-(trimethoxysilyl)propyl methacrylate at a temperature of $120^{\circ} \mathrm{C}$ for 6 hours. In order to avoid the spontaneous polymerization of the functional methacrylate, a stable free radical (DPPH) was added to the solution. The SEM micrographs of Figure 8 show that the resulting monolith exhibits a "classical" macroporous structure "wrapped" in a thin outer layer of apparently nonporous polymer.

The reactive chloromethyl moieties incorporated into the monolith served as sites for the introduction of quaternary ammonium functionalities according to the Scheme 1. The pores of the monolith were filled with $N, N$-dimethyloctylamine, and after a suitable reaction period, the column was washed with methanol and equilibrated with the mobile phase. Unfortunately, only very limited information concerning the nature and extent of modification was presented.

These capillary columns possessing positively charged surface functionalities were used for the reversed-phase separations of basic and acidic peptides. Figure 9 shows the excellent separation of three angiotensins and insulin with plate numbers as high as $200,000 \mathrm{pl} / \mathrm{m}$ using acetonitrile and phosphate buffer $(\mathrm{pH}=3)$. Surprisingly, the retentions of both angiotensins I and II increased as the percentage of acetonitrile in the mobile phase was increased from 25 to $45 \%$, and no elution was observed at higher percentages of organic solvent. 


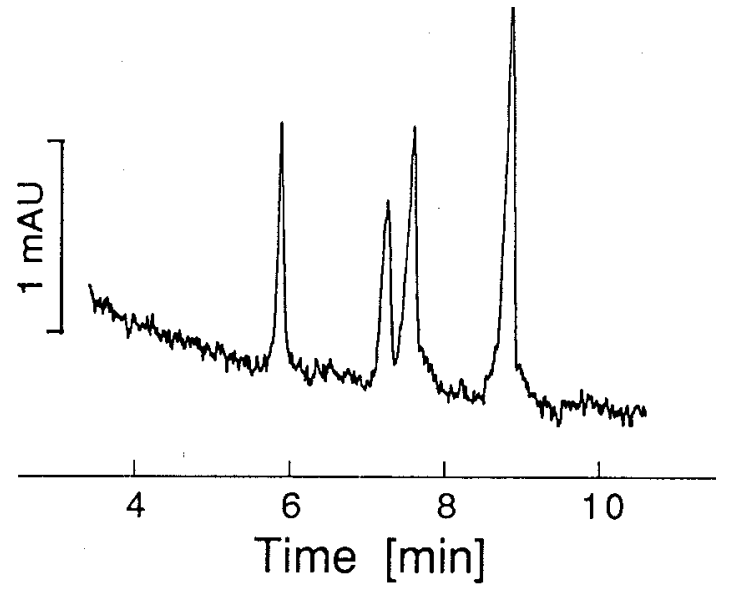

Figure 10. Electrochromatogram of four basic proteins obtained by isocratic separation using a modified polychloromethylstyrene-based PLOT column (Reprinted with permission from ref. 29. Copyright 1999 Elsevier). Column $47 \mathrm{~cm}$ (active length $40 \mathrm{~cm}$ ) $\times 20 \mu \mathrm{m}$, inner polymer layer $2 \mu \mathrm{m}$; mobile phase $20 \%$ acetonitrile in $20 \mathrm{mmol} / \mathrm{l}$ phosphate buffer $\mathrm{pH} 2.5$; voltage $-30 \mathrm{kV}$; EOF velocity measured with dimethylsulfoxide (DMSO) $-3.46 \times 10^{-8} \mathrm{~m}^{2} \mathrm{~V}^{-1} \mathrm{~s}^{-1}$, migration time for DMSO $3.10 \mathrm{~min}$. Peaks: $\alpha$-chymotripsinogen (1), ribonuclease (2), cytochrome C (3), lysozyme (4).

Good separation of chemically similar tripeptides (Gly-GlyPhe and Phe-Gly-Gly) was also observed in a pH 7 buffer using unfunctionalized poly(styrene-co-divinylbenzene) monoliths devoid of charged functionalities. In this case, the driving force for movement of the analytes through the column is their electrophoretic migration, while separation results from their interactions with the stationary phase [28]. However, the addition of acetonitrile to the mobile phase significantly decreases the analyte mobility, making this approach less attractive.

Horváth et al. also produced macroporous polymer layer open tubular capillary columns (PLOT) for CEC similar to those employed in gas chromatography [29]. Narrow bore capillaries $(20 \mu \mathrm{m}$ i.d.) are required in order to achieve high efficiency. The preparation process is closely related to that described previously. A vinylized capillary was filled with a polymerization solution of chloromethylstyrene, divinylbenzene, and 2-octanol as a porogen, and an in situ polymerization was performed. The resulting porous polymer layer was functionalized by reaction with $N, N$-dimethyloctadecylamine and any residual chloromethyl groups quenched by reaction with a solution of sodium hydroxide. Although no experimental data concerning the extent of functionalizations or process were published, the surface coverage of positively charged functionalities was sufficiently large to afford EOF velocities of $2.1-2.5 \mathrm{~mm} / \mathrm{s}$ that did not change significantly over the broad range of $0-60 \%$ acetonitrile in the mobile phase.

Horvath also demonstrated the complex nature of CEC separations that often involve the interplay of EOF, electrophoretic migration, and chromatographic retention through several examples of peptide and protein separations using the PLOT capillary columns. For example, the retention of proteins was seen to increase with increasing percentage of organic solvent in the mobile phase. Even more intriguing is the demonstrated isocratic separation of proteins shown in Figure 10. Although the window of mobile phase compositions within which this separation could be achieved was narrow, this approach deserves further study since it may eliminate the need for the rather complex gradient elution instrumentation.

\section{Methacrylate Ester-Based Monolithic Columns}

In contrast to the reported characterizations of the acrylamide and styrene-based monoliths that have largely been limited to evaluation of their chromatographic performances, extensive materials development and optimization have been performed for monolithic CEC capillaries prepared from methacrylate ester monomers. These investigations made use of the concepts developed from our original work with the molded rigid monolithic HPLC columns we introduced in the early 1990s [30, 31]. The experience acquired earlier with these materials proved helpful in investigating the interrelated effects of morphology and composition on the overall CEC process.

Production of these monolithic capillary columns is amazingly simple [32]. Either a bare or a surface treated capillary is filled with a homogeneous polymerization mixture, and radical polymerization is initiated only when desired using either a thermostated bath or UV irradiation [33] to afford a rigid monolithic porous polymer. Once the polymerization is complete, unreacted components such as the porogenic solvents are removed from the monolith using a syringe pump or electroosmotic flow (Figure 1). This simple method for preparing monolithic capillary columns has numerous advantages. For example, the fused silica tubing may be used either directly as supplied without first performing any chemical modification of its internal surface or after its functionalization using a suitable vinyl containing moiety. All of the chemicals may be used as supplied, although careful purification contributes to better batch-to-batch reproducibility (vide infra). Additionally, the final polymerization mixture contains free radical initiators such as benzoyl peroxide or azobisisobutyronitrile, ensuring its stability and easy handling for several hours at room temperature or for days in the refrigerator without risking the onset of polymerization.

In optimizing the process, specific attention was paid to the design of the porogenic mixtures. Ideally, this system had to enable (i) the preparation of a homogeneous, single phase polymerization mixture from the charged, water soluble monomer that supports the EOF, and the hydrophobic monomers that affect the separation without using additional compatibilizing agents; (ii) the direct uniform incorporation of these monomers with widely differing polarities into a macroporous polymer monolith; (iii) the fine control of the 


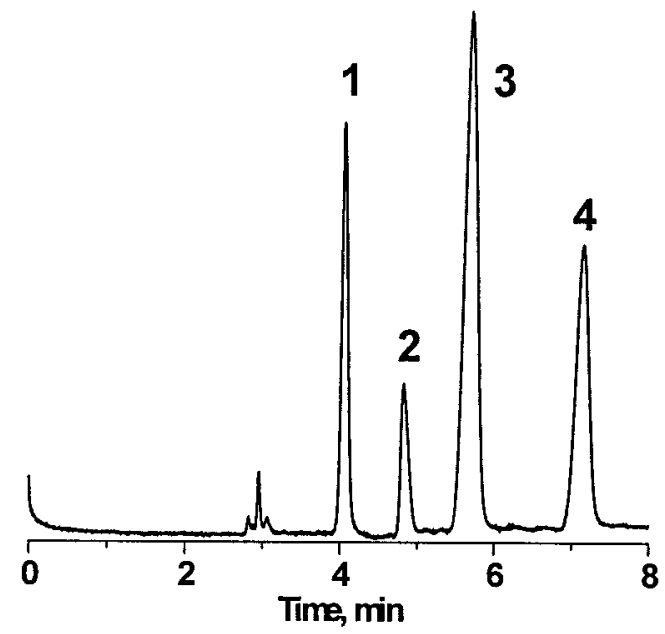

Figure 11. Electrochromatographic separation of Gly-Tyr (1), Val-TyrVal (2), methionine enkephalin (3), and leucine enkephalin (4) on monolithic capillary column $100 \mu \mathrm{m}$ i. d. $\times 28 \mathrm{~cm}$ with a pore size of $492 \mathrm{~nm}$. Conditions: Mobile phase $80 \%$ of a 1:9 mixture of $10 \mathrm{mmol} / \mathrm{L}$ sodium 1octanesulfonate and $5 \mathrm{mmol} / \mathrm{L}$ phosphate buffer $\mathrm{pH}=7.0$, and $20 \%$ acetonitrile. UV detection at $215 \mathrm{~nm}$. Total sample concentration $1 \mathrm{mg} / \mathrm{mL}$.

porous properties of the resulting monolith over a broad range; and finally, (vi) the facile initial washing and equilibration of the capillary column by being miscible with the mobile phase used for electrochromatography. An extensive study led to the development of a ternary porogen system consisting of water, 1-propanol, and 1,4-butanediol in various proportions [32]. Monolithic capillary columns prepared using this porogen system and photochemical initiation possessed efficiencies of over $210000 \mathrm{pl} / \mathrm{m}$ for the separation of a model mixture of aromatic compounds [33]. Similarly, peptides were separated on this capillary column using a mobile phase containing 1-octanesulfonic acid (Figure 11). This ionpairing alkylsulfonic acid additive likely affects the separation of the peptides both by associating with the terminal amine groups of the peptides and preventing them from interacting with the negatively charged surface functionalities of the monolith as well as by increasing the hydrophobicity of the analytes [33].

The methacrylate-based polymers are stable even under extreme $\mathrm{pH}$ conditions such as $\mathrm{pH} 2$ or 12 [34]. The sulfonic acid functionalities of the monolithic polymer remain dissociated over this entire $\mathrm{pH}$ range creating a flow velocity sufficient to achieve the separations in a short period of time. In contrast to the stationary phase, the analytes are uncharged, yielding symmetrical peaks. It should be noted that such extreme $\mathrm{pH}$ conditions cannot be tolerated by typical silicabased packings.

\section{Reproducibility of Monolithic Columns for CEC}

Although the reproducible preparation and operation of CEC columns are extremely important issues that will further sti-
Table 1. Reproducibility of the electrochromatographic properties of methacrylate-based monolithic capillaries. Conditions: capillary columns, $100 \mu \mathrm{m}$ i.d. $\times 30 \mathrm{~cm}$ active length; stationary phase poly(butyl methacrylate-co-ethylene dimethacrylate) with $0.3 \mathrm{wt} \%$ 2-acrylamido-2methyl-1-propanesulfonic acid; mobile phase, 80:20 vol./vol. mixture of acetonitrile and $5 \mathrm{mmol} / \mathrm{L}$ phosphate buffer $\mathrm{pH} 7$; UV detection at $215 \mathrm{~nm}$; voltage $25 \mathrm{kV}$; pressure in vials $0.2 \mathrm{MPa}$; sample concentration $2 \mathrm{mg} / \mathrm{mL}$ of each compound; injection $5 \mathrm{kV}$ for $3 \mathrm{~s}$. Data shown are average RSD values obtained for thiourea, benzyl alcohol, benzaldehyde, benzene, toluene, ethylbenzene, propylbenzene, butylbenzene, and amylbenzene.

\begin{tabular}{lccc}
\hline Variable & \multicolumn{3}{c}{ RSD\% } \\
\cline { 2 - 4 } & $\begin{array}{c}\text { Run-to-run } \\
n=14\end{array}$ & $\begin{array}{c}\text { Day-to-day } \\
n=3\end{array}$ & $\begin{array}{c}\text { Column-to-column } \\
n=7\end{array}$ \\
\hline Retention time & 0.18 & 1.19 & 3.50 \\
Retention factor & 0.21 & 0.30 & 1.43 \\
Selectivity & 0.05 & 0.10 & 0.11 \\
Efficiency & 1.50 & 4.30 & 7.80 \\
\hline
\end{tabular}

mulate the development and the acceptance of this technique, only a few groups have reported data on column-to-column, run-to-run, and day-to-day reproducibility of monolithic capillary columns. Novotny showed reproducibility data for migration times $t_{r}$, efficiencies, and retention factors $k^{\prime}$ for a number of analytes on acrylamide-based monoliths [16]. The relative standard deviations (RSD) were smaller for run-torun compared to day-to-day measurements. For example, the average run-to-run RSD for 6 analytes was $0.8 \%$ for $t_{r}, 2.6 \%$ for $k^{\prime}$, and $4.3 \%$ for the efficiency, while the average day-today RSDs for the same variables were $2.1 \%, 6.1 \%$, and $4.6 \%$, respectively.

Horvath monitored the conductivity of his modified monolithic polystyrene-based columns for over 3 months and observed no changes [28]. Similarly, the electroosmotic mobility was measured over a number of days and again almost no changes were found [29].

Tests of the reproducibility of retention times, retention factors, separation selectivities, and column efficiencies for our methacrylate monolithic capillary columns are summarized in Table 1. This table shows averaged data obtained for 9 different analytes injected 14 times repeatedly every other day over a period of 6 days, as well as for 7 different capillary columns prepared from the same polymerization mixture. As expected, both injection-to-injection and day-to-day reproducibilities measured for the same column are very good. Slightly larger RSD values were observed for column-to-column reproducibility. While the selectivity effectively did not change, larger differences were found for the efficiencies of the columns. Unfortunately, no reproducibility data are currently available for any of the acrylamide gel-based continuous beds prepared from aqueous solutions. 


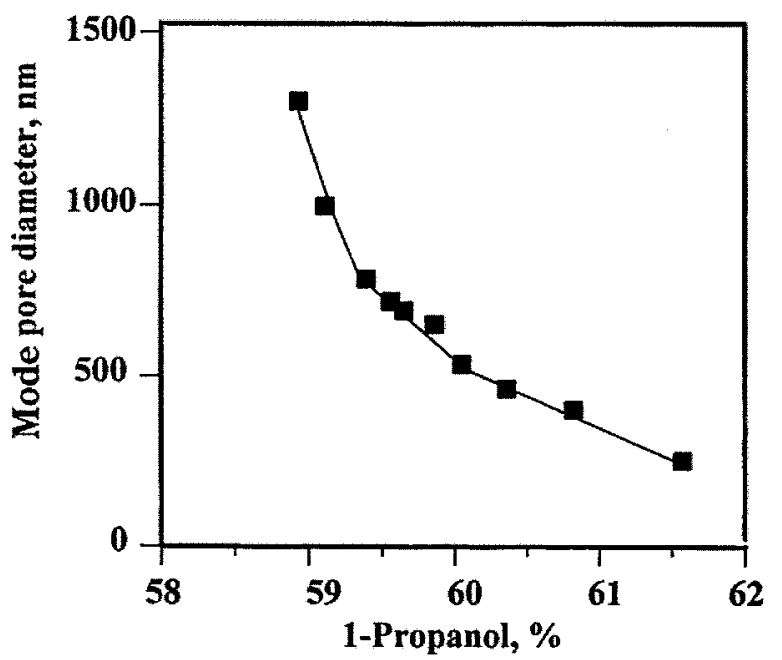

Figure 12. Effect of the percentage of 1-propanol in the porogenic mixture on the porous properties of monolithic polymers (Reprinted with permission from ref. 37. Copyright 1998 American Chemical Society). Reaction conditions: polymerization mixture: ethylene dimethacrylate 16.00 wt. \%, butyl methacrylate 23.88 wt. $\%$, 2-acrylamido-2-methyl-1propanesulfonic acid 0.12 wt. $\%$, ternary porogen solvent 60.00 wt. $\%$ (consisting of $10 \mathrm{wt} . \%$ water and $90 \mathrm{wt} . \%$ of mixtures of 1-propanol and 1,4-butanediol), azobisisobutyronitrile 1 wt.\% (with respect to monomers), polymerization time $20 \mathrm{~h}$ at $60^{\circ} \mathrm{C}$.

\section{Assessment of the Porous Structure}

The ability of a liquid to flow through the network of channel-like pores that traverse the length of these monolithic materials is essential to all of their applications. In addition to providing permeability, the porous structure also accelerates the rate of mass transfer within the separation medium as a result of convection [35], since all of the mobile phase flows through the pores [36]. Despite this important fact, only one study directly assessing the effect of pore size of the monolithic CEC media has been published [37]. The absence of data for other monolithic systems is probably due both to the limited means available to control their porous structures during preparation as well as to difficulties in determining their actual pore structure in the swollen state. It should be emphasized that the standard methods typically used for the measurement of porous properties such as mercury intrusion porosimetry and nitrogen absorption/desorption methods, are performed on materials in the dry state, while the columns actually operate in the presence of a solvent. As a result, the data measured in the dry state may not actually reflect the operational pore size of the capillaries during the actual chromatographic process.

In a recent study, Horváth used three methods to determine the porosity of monolithic capillary columns in the "solvated" state [28]. First, the elution time of a low molecular nonretained tracer in $\mu$ HPLC was used to calculate the total porosity. The second method afforded an estimation of the porosity from the conductivity ratio. Monolithic and empty capil-

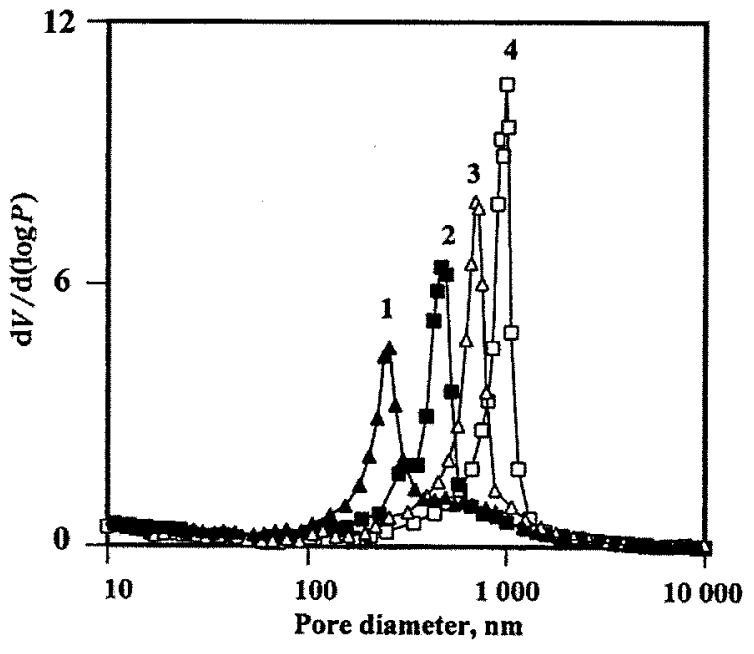

Figure 13. Differential pore size distribution profiles of porous polymeric monolithic capillary columns with mode pore diameters of 255 (curve 1), 465 (2), 690 (3), and $1000 \mathrm{~nm}$ (4) (Reprinted with permission from ref. 37. Copyright 1998 American Chemical Society).

laries were filled with an electrolyte and their conductivities were measured. Although several equations relating conductivity ratio to total column porosity have been derived, Archie's equation appeared to provide the best fit of the experimental data. The last method was gravimetric, using the weight difference between a dry and acetone filled monolithic column. Since none of these three methods affords information about pore size distribution, liquid extrusion porosimetry with hexadecane was used to determine the integral pore volume distribution. However, since this technique requires samples larger than those available from a capillary column, it was performed using a sample prepared via a larger scale bulk polymerization [28]. Similarly, we polymerized the same mixture used for the preparation of capillary columns in glass vials and used the product for mercury intrusion porosimetry. Since we found that a strong correlation exists between the "dry" porous properties of the monoliths and their chromatographic performance, even "dry" porosity measurements may be used to tailor column performance.

The novel ternary porogenic system that we have developed enables precise control of porous properties over a broad range [37]. For example, the percentage of 1-propanol in the porogenic solvent exerts an enormous effect on the pore diameter at the maximum of the distribution curve (mode pore diameter) as documented in Figure 12 for the UV initiated polymerization system. Based on these results, monoliths of any pore size within the broad range of $250-1300 \mathrm{~nm}$ can easily be produced by simply changing the ratio of propanol to butanediol in the porogenic mixture. It should be noted that the window of weight percentage of 1-propanol that brackets this wide range of pore sizes is sufficiently large to obtain polymers of any mode pore diameter with an accuracy of $\pm 25 \mathrm{~nm}$ with respect to the targeted value. Despite the fact 


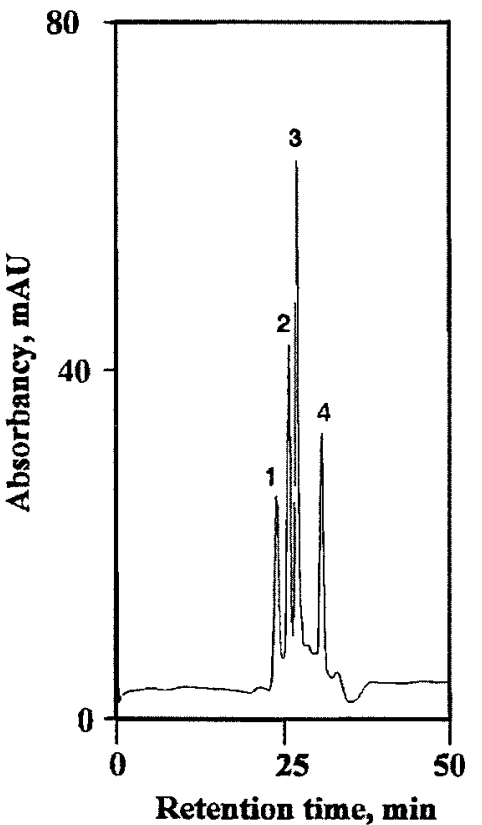

Figure 14. Electrochromatographic size-exclusion chromatography of polystyrene standards (Reprinted with permission from ref. 37. Copyright 1998 American Chemical Society). Conditions: monolithic capillary column, $100 \mu \mathrm{m}$ i.d. $\times 30 \mathrm{~cm}$ active length; stationary phase, $59.7 \mathrm{wt} . \%$ butyl methacrylate, and $40 \mathrm{wt} \%$ ethylene dimethacrylate in monomer mixture, 0.3 wt.\% 2-acrylamido-2-methyl-1-propanesulfonic acid; pore size $750 \mathrm{~nm}$; mobile phase, tetrahydrofuran containing 2 vol.\% of water; $\mathrm{UV}$ detection at $215 \mathrm{~nm}$; voltage, $25 \mathrm{kV}$; pressure in vials, $0.2 \mathrm{MPa}$; sample concentration, $2 \mathrm{mg} / \mathrm{mL}$ of each compound; injection, $5 \mathrm{kV}$ for $3 \mathrm{~s}$; peaks, polystyrene standards, molecular weight 980,000 (1), 34,500 (2), 7,000 (3), and toluene (4).

that these monoliths are prepared from a polymerization mixture containing monomers of very different polarities, all of the mercury porosimetry profiles seen in Figure $\mathbf{1 3}$ exhibit distribution curves similar to those found for polymers prepared from mixtures of fully miscible monomers [38], as well as the CEC systems described initially [37].

Such precise control of porous properties is expected to be very useful in the design of specialized CEC columns for separation in modes other than reversed-phase. For example, size exclusion chromatography (SEC) is an isocratic separation method that relies on differences in the hydrodynamic volumes of the analytes. Because all solute-stationary phase interactions must be avoided in SEC, solvents such as pure THF are often used as the mobile phase for the analysis of synthetic polymers, since they dissolve a wide range of structures and minimize interactions with the chromatographic medium. Despite the reported use of entirely non-aqueous eluents in both electrophoresis and CEC [39], no appreciable flow through the methacrylate-based monoliths was observed using pure THF as the mobile phase. However, a mixture of $2 \%$ water in THF was found to substantially accelerate the

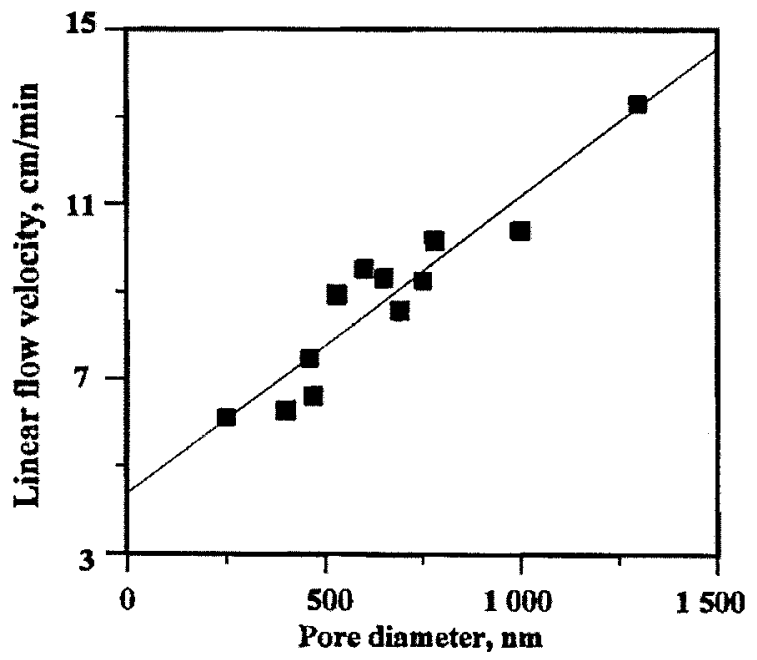

Figure 15. Effect of mode pore diameter on flow velocity of the mobile phase through monolithic capillary columns (Reprinted with permission from ref. 37. Copyright 1998 American Chemical Society). Conditions: capillary column, $100 \mu \mathrm{m}$ i.d. $\times 30 \mathrm{~cm}$ active length; stationary phase with $0.3 \mathrm{wt} \% \%$ 2-acrylamido-2-methyl-1-propanesulfonic acid; pore size, $465 \mathrm{~nm}$; mobile phase, $80: 20$ vol./vol mixture of acetonitrile and $5 \mathrm{mmol} / \mathrm{L}$ phosphate buffer $\mathrm{pH} 7$.

flow velocity, while still capable of dissolving polystyrene standards with molecular weights as high as 980000 [40]. Figure 14 shows the first SEC separation of polystyrenes in the CEC mode using a methacrylate-based monolithic capillary column. The molecular weights of the peaks were assigned by injections of the individual standards. The elution order of the polystyrene standards and toluene confirms that size exclusion is the prevailing separation mechanism. Although the porous properties of the monolithic column used for this experiment were not optimized for SEC separations, these results demonstrate that CEC is not limited to the reversed-phase mode of chromatography. An extensive study of SEC separations of polystyrenes in the CEC mode using packed capillary columns and dimethylformamide as the solvent has recently been published [41].

\section{Effects of the Properties of the Monoliths on the Separation in CEC}

The ability to achieve precise and independent control over both the porous properties as well as the level of charged moieties of the rigid monolithic stationary phases opened new avenues for studies focusing on the effects these properties exert on the chromatographic process. Both of these variables were found to be extremely important in controlling the flow velocity and efficiency of the monolithic capillary CEC columns. In addition to these materials properties, CEC separations are also affected by the conditions under which they are performed, including the applied voltage, and both the $\mathrm{pH}$ and elution strength of the mobile phase. 


\subsection{Pore Size and Efficiency}

The major advantage of CEC compared to classical HPLC is that much higher column efficiencies can be achieved using identical separation media. For columns packed with beads, the efficiency of both of these methods is particle size dependent, and increases as the size of the packing decreases [7]. Since the monolithic columns are molded rather than packed, issues of particles size become irrelevant, and instead the size of the pores within the monolithic material is the variable most expected to affect chromatographic efficiency. Indeed, initial studies have shown that the size of the flow-through pores dramatically affects separation efficiency [32, 37]. However, having defined preparative parameters that yield capillaries possessing acceptable efficiencies, this effect becomes rather complex, and its subtleties remain unexplored.

\subsection{Solvent Flow through the Monolithic Capillary}

Electroosmotic flow is generally reported to be independent of the size of the packing, and consequently the size of the interstitial voids between the particles, unless this size is so small that the electrical double layers overlap [42]. The ability to independently control both the pore size and level of charged functionalities of the methacrylate ester monolithic capillaries enables the direct investigation of the net effect of transport channel size on flow velocity. Figure 15 clearly demonstrates a two-fold increase in flow velocity through the thermally initiated monolithic capillaries with the same level of charged moieties as the pore size increases from $250 \mathrm{~nm}$ to $4 \mu \mathrm{m}$ [37]. A similar increase in flow velocity was observed for monoliths prepared by UV initiated polymerization [33]. This range of pore sizes significantly exceeds the thickness of a few nanometers at which the electrical double layers would overlap for a system utilizing a mobile phase containing $5 \mathrm{mmol} / \mathrm{l}$ buffer [37]. If it is assumed that the observed decrease in flow rate with decreasing pore diameter simply results from the increasing percentage of pores within which overlap of the electric double layers occurs, then the flow velocity should reach a maximum value for those monoliths having sufficiently large pores, and remain constant thereafter, since the number of pores within which overlap of the electric double layer can occur decreases rapidly as the pore size increases. In practice however, this phenomenon is not observed. The fact that the overall flow velocity increases linearly over a broad range of pore sizes may support the contention that this increase in flow rate is macroscopically related to a decrease in the resistance to flow through the channels. An additional effect may result from microscopic variations in the strength of the electrical field in both the small and large pores. The effects of tortuosity and variations in the cross sectional area of a packed structure on the conductance and chromatographic performance of CEC capillaries packed with beads have recently been discussed in the literature [43].

\subsection{Control of Surface Chemistry}

Electroosmotic flow velocity is directly proportional to the zeta potential, that in turn is directly related to the surface charge. In contrast to silica-based CEC media, the ability to easily control the level of charged functionalities that support the electroosmotic flow is a major advantage of the polymeric monolithic capillaries. This variable can easily be adjusted by changing the percentage of charged monomer in the polymerization mixture. For example, linear increases in migration velocity paralleling increases in AMPS monomer content were observed by Fujimoto in both $6 \%$ crosslinked polyacrylamide gels [10] as well as $9.7 \%$ crosslinked $N$-isopropylacrylamide polymers [11]. Similarly, increasing the content of sulfonic acid groups within the methacrylate ester monoliths significantly increased the flow velocity, thus reducing the overall analysis time. Similar chromatographic performances were maintained in these higher flow capillaries by making concomitant changes in the composition of the porogenic mixture in order to keep the pore sizes of the monoliths effectively constant.

Monoliths containing two significantly different percentages of dimethyldiallylammonium chloride were recently prepared in order to control the EOF component of the overall migration rate of proteins [15]. These charged moieties were incorporated into the monolith during a later stage of the preparation process, and appeared to be well suited for the desired separation (vide supra).

\subsection{Retention and Selectivity}

The majority of CEC separations reported to date have been performed in the reversed-phase mode. Under these conditions, the hydrophobicity of the stationary phase determines the selectivity of the separation, and retention can easily be controlled by adjusting either the composition of the mobile phase or the hydrophobicity of the surface, with the first option being easier to implement. However, in contrast to the rich variety of solvents available for use in HPLC, acetonitrile-based solvent systems are employed in most CEC applications due to their high dielectric constant and low viscosity $[14,16,37,44]$.

The effect of surface polarity is enhanced in separations where two or more simultaneous interactions must occur in order to achieve the desired selectivity. This is particularly true in molecular recognition processes such as chiral separations. Since aqueous buffer systems are almost universally used as CEC mobile phases, enantioseparations are often run under reversed-phase conditions as opposed to the normalphase mode typically used in chiral HPLC. Therefore, nonspecific hydrophobic interactions would be highly detrimental to the discrimination process that involves subtle differences between the enantiomers.

The importance of tailoring surface chemistry is demonstrated by three different monolithic capillary columns that were pre- 


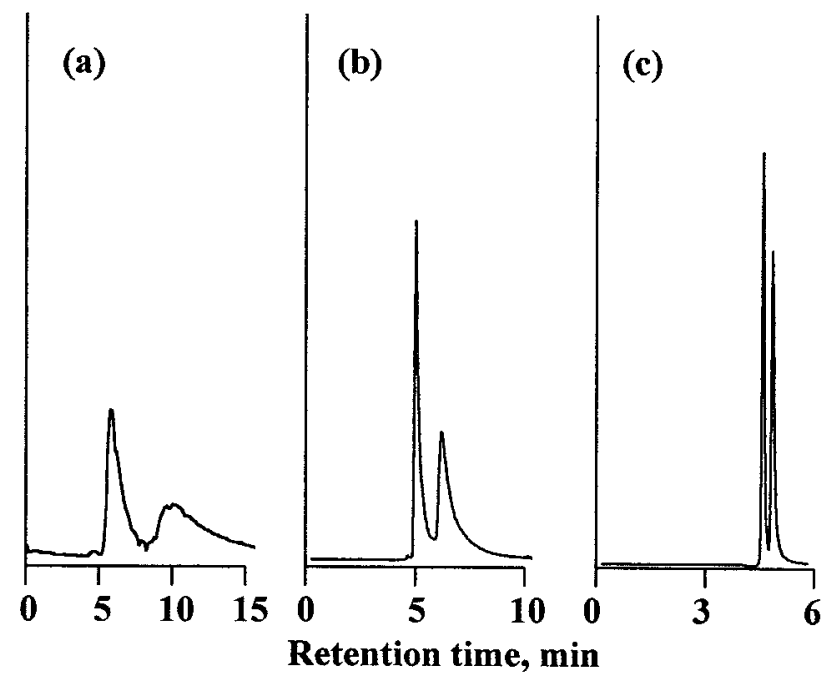

Figure 16. Effect of the hydrophilicity of chiral monolithic columns on the electrochromatographic separation of $N$-(3,5-dinitrobenzoyl)leucine diallylamide enantiomers (Modified from ref. 45). Conditions: monolithic column, $100 \mu \mathrm{m}$ i.d. $\times 30 \mathrm{~cm}$ active length; mobile phase, 80:20 vol./vol. mixture of acetonitrile and $5 \mathrm{mmol} / \mathrm{L}$ phosphate buffer $\mathrm{pH} 7$; $\mathrm{UV}$ detection at $215 \mathrm{~nm}$; voltage, $25 \mathrm{kV}$; pressure in vials, $0.2 \mathrm{MPa}$; injection, $5 \mathrm{kV}$ for $3 \mathrm{~s}$. Stationary phase with butyl methacrylate (a), glycidyl methacrylate (b), and hydrolyzed glycidyl methacrylate (c).

pared by directly incorporating of the chiral monomer 2hydroxyethyl methacrylate ( $N$-L-valine-3,5-dimethylanilide) carbamate [45]. These columns were tested for the enantioseparation of a model racemic compound, $N$-(dinitrobenzoyl)leucine diallylamide. Figure 16 compares the chiral separations achieved using the various columns. Although the column containing butyl methacrylate as a hydrophobic comonomer did resolve the racemic analyte, the peaks were very broad and tailed severely. The efficiency of this system was poor, with plate counts of only 600 and 160 plates/m obtained for the respective enantiomers (Figure 16.a). However, when pure acetonitrile was used as the mobile phase, a narrowing of both peaks and a concomitant increase in column efficiency to 2,500 and 540 plates/m were observed. These improvements indicate that the originally observed tailing probably resulted from non-specific hydrophobic interactions between the chiral analyte and the relatively hydrophobic surface of the separation medium. Therefore, new capillary columns were prepared substituting the more hydrophilic glycidyl methacrylate for the highly hydrophobic butyl methacrylate. Although this capillary column exhibited a surprisingly high efficiency of 210,000 plates $/ \mathrm{m}$ for the unretained peak of thiourea under reversed-phase conditions, the incorporation of the more hydrophilic monomer resulted in a substantial decrease in hydrophobic selectivity $\left(\alpha_{\mathrm{CH}_{2}}=1.08\right)$. This change in surface polarity resulted in a significantly improved chiral separation (Figure 16.b). The peaks for the enantiomers were sharper, and the column efficiencies calculated for this separation increased to 8,100 and 1,900 plates $/ \mathrm{m}$.
Instead of defining and optimizing new conditions for the direct incorporation of an even more hydrophilic monomer into the monolith, the epoxide rings of the monolith described above that contains glycidyl methacrylate and chiral units were hydrolyzed using dilute aqueous sulfuric acid to afford very hydrophilic diol functionalities. This hydrolytic reaction was easily performed in situ within the pores of the monolithic capillary column. After hydrolysis, the diol-functionalized hydrophilic capillary was unable to effect any separation of alkylbenzenes in the reversed-phase mode. However, this monolithic column afforded a significantly improved separation of the enantiomers (Figure 16.c). The peaks in this separation were narrow and well resolved $\left(R_{\mathrm{s}}=2.0\right)$. Column efficiencies $(61,000$ and 49,500 plates/m) were rather high, and even peak tailing was greatly reduced, suggesting that few undesirable interactions remained. Unfortunately, this substantial increase in column efficiency was accompanied by a decrease in selectivity.

This example clearly demonstrates the benefits of the facile tuning of surface chemistry afforded by the monolithic media. The wealth of commercially available monomers possessing a variety of functionalities, together with the extreme simplicity of the preparation of the monolithic columns, makes this approach an appealing option for the design of capillary columns with high selectivities.

\section{Conclusion}

Monolithic stationary phases have emerged as attractive alternatives to packed CEC columns due to the simplicity of their preparation as well as the virtually unlimited choice of chemistries they offer. This technology completely eliminates the need for retaining frits and their associated problems, thus decreasing the technical barriers to the general acceptance of the CEC technique. In contrast to packed beds, monolithic structures exhibit excellent dimensional stability as a result of their rigidity and/or chemical attachment to the inner wall of the capillary.

Monolithic materials can easily be prepared even within channels of very narrow dimensions by a single step in situ polymerization, and the formation of the monolith can be restricted to a specific area using photochemically initiated polymerization. This suggests that this technology may uniquely be suited for the further development of miniaturized analytical systems on-chip.

The number of different approaches to monolithic columns for CEC published in the literature and under development in both academic and industrial laboratories is growing rapidly. This review only summarized the details of monolithic CEC columns prepared from synthetic polymers. However, analogous approaches resulting in entirely inorganic-based monolithic capillary columns prepared by direct condensation of silicon oxide [46-48], entrapping of inorganic particles in 
inorganic gels [49-52], fusing [53], and sintering of silica beads $[54,55]$ have emerged recently.

Hybrid methods and alternative column technologies have also been described, including the immobilization of molecularly imprinted particles within a polymer gel matrix $[55,56]$, the use of in situ prepared polymer monoliths as retaining frits for packed beds [57], and "hanging" cellulose acetate fibers anchored at one end in the porous silicate inlet frit [58].

Although much remains to be done in the development of stationary phases for CEC, the monolithic columns appear to be a very promising technology that specifically addresses many of the unique aspects of CEC. Among their numerous advantages, their reproducibility, variety of well defined chemistries, high efficiencies, and ease of preparation are expected to further accelerate the development of CEC, and lead to novel columns for the separations of compounds from complex matrices in a variety of chromatographic modes.

\section{Acknowledgment}

Support of this research by a grant of the National Institute of General Medical Sciences, National Institutes of Health (GM-48364) is gratefully acknowledged. This work was also supported by the Division of Materials Sciences of the U.S. Department of Energy under Contract No. DEAC03-76SF00098.

\section{References}

[1] V. Pretorius, B.J. Hopkins, J.D. Schieke, J. Chromatogr. 1974, 99 , 23.

[2] J.W. Jorgenson, K.D. Lukacs, J. Chromatogr.1981, 218, 209.

[3] T. Tsuda, K. Nomura, G. Nakagawa, J. Chromatogr. 1982, 248, 241.

[4] J.H. Knox, I.H. Grant, Chromatographia 1987, 24, 135.

[5] B. Boughtflower, T. Underwood, Chromatographia 1995, 38, 329.

[6] K. Schmeer, B. Behnke, E. Bayer, Anal. Chem. 1995, 67, 3656.

[7] J.H. Knox, J. Chromatogr. 1994, 680, 3.

[8] Y. Baba, M. Tsuhako, Trends Anal. Chem. 1992, 11, 280.

[9] C. Fujimoto, J. Kino, H. Sawada, J. Chromatogr. A 1995, 716, 107.

[10] C. Fujimoto, Anal. Chem. 1995, 67, 2050.

[11] C. Fujimoto, Analusis 1998, 16, M49.

[12] S. Hjertén, D. Eaker, K. Elenbring, C. Ericson, K. Kubo, J.-L. Liao, C.-M. Zeng, P.-A. Lidström, C. Lindh, A. Palm, T. Srichiayo, L. Valtcheva, R. Zhang, Jpn. J. Electrophor. 1995, 39, 105.

[13] C. Ericson, J.L. Liao, K. Nakazato, S. Hjertén J. Chromatogr. A 1997, 767, 33.

[14] J.L. Liao, N. Chen, C. Ericson, S. Hjertén, Anal. Chem. 1996, 68, 3468 .

[15] C. Ericson, S. Hjertén, Anal. Chem. 1999, 71, 1621.
[16] A. Palm, M.V. Novotny, Anal. Chem. 1997, 69, 4499.

[17] P.G. Righetti, J. Chromatogr. A 1995, 698, 3.

[18] G. Wulff, Angew. Chem. Int. Ed. Engl. 1995, 34, 1812.

[19] K.J. Shea, Trends Polym. Sci. 1994, 2, 166.

[20] K. Mosbach, Trends Biochem. Sci. 1994, 19, 9.

[21] S. Nilsson, L. Schweitz, M. Petersson, Electrophoresis 1997, 18, 884.

[22] L. Schweitz, L.I. Andersson, S. Nilsson, J. Chromatogr. A 1997, $792,401$.

[23] L. Schweitz, L.I. Andersson, S. Nilsson, Anal. Chem. 1997, 69, 1179.

[24] J.M. Lin, T. Nakagama, X.Z. Wu, K. Uchiyama, T. Hobo, Fres. J. Anal. Chem. 1997, 357, 130.

[25] J.M. Lin, T. Nakagama, K. Uchiyama, T. Hobo, Chromatographia 1996, 43, 585.

[26] L. Schweitz, L.I. Andersson, S. Nilsson, J. Chromatogr. A 1998, 817,5 .

[27] T. Koide, K. Ueno, Anal. Sci. 1998, 14, 1021.

[28] I. Gusev, X. Huang, C. Horváth, J. Chromatogr. A 1999, 855, 273.

[29] X. Huang, J. Zhang, C. Horváth, J. Chromatogr. A 1999, 858, 91.

[30] F. Svec, J. M. J. Fréchet, Anal. Chem. 1992, 64, 820.

[31] F. Svec, J. M. J. Fréchet, Science 1996, 273, 205.

[32] E.C. Peters, M. Petro, F. Svec, J.M.J. Fréchet, Anal. Chem. 1997, $69,3646$.

[33] C. Yu, F. Svec, J. M. J. Fréchet, Electrophoresis 2000, 21, 120.

[34] F. Svec, E.C. Peters, D. Sýkora, J.M.J. Fréchet, J. Chromatogr. A, in press.

[35] F.E. Regnier, Nature 1991, 350, 643.

[36] D.K. Roper, E.N. Lightfoot, J. Chromatogr. A 1995, 702, 3.

[37] E.C. Peters, M. Petro, F. Svec, J.M.J. Fréchet, Anal. Chem. 1998, $70,2288$.

[38] C. Viklund, F. Svec, J.M.J. Fréchet, K. Irgum, Chem. Mater. 1996, 8,744 .

[39] E.C. Peters, M. Petro, F. Svec, J.M.J. Fréchet, Anal. Chem. 1998, 70, 2296.

[40] P.B. Wright, A.S. Lister, J.G. Dorsey, Anal. Chem. 1997, 69, 3251.

[41] E. Venema, J.C. Kraak, H. Poppe, R. Tijssen, Chromatographia $1998,48,347$.

[42] J.H. Knox, I.H. Grant, Chromatographia 1991, 32, 317.

[43] G. Choudhary, C. Horváth, J. Chromatogr. A 1997,781, 161.

[44] C. Fujimoto, Y. Fujise, E. Matsuzawa, Anal. Chem. 1996, 68, 2753.

[45] E.C. Peters, K. Lewandowski, M. Petro, F. Svec, J.M.J. Fréchet, Anal. Commun. 1998, 35, 83.

[46] S.M. Fields, Anal. Chem. 1996, 68, 2709. 
[47] H. Minakuchi, K. Nakanishi, N. Soga, N. Ishizuka, N. Tanaka, Anal. Chem. 1996, 68, 3498.

[48] N. Ishizuka, H. Minakuchi, K. Nakanishi, N. Soga, K. Hosoya, N. Tanaka, J. High Resol. Chromatog. 1998, 21, 477.

[49] M.T. Dulay, R.P. Kulkarni, R.N. Zare, Anal. Chem. 1998, 70, 5103.

[50] G. Chirica, V.T. Remcho, Electrophoresis 1999, 20, 50.

[51] Q. Tang, B. Xin, M.L. Lee, J. Chromatogr. A, 1999, 837, 35.

[52] S.A. Rodriguez, L.A. Colón, Chem. Mater. 1999, 11, 754.
[53] M.M. Dittmann, G.P. Rozing, G. Ross, T. Adam, K.K. Unger, $J$. Capill. Electrotrophoresis 1997, 4, 201.

[54] R. Asiaie, X. Huang, D. Farnan, C. Horváth, J. Chromatogr. A 1998, 806, 251.

[55] D. Li, V.T. Remcho, J. Microcol. Sep. 1997, 9, 389.

[56] J.M. Lin, T. Nakagama, K. Uchiyama, T. Hobo, Chromatographia 1996, $43,585$.

[57] J.R. Chen, R.N. Zare, Anal. Chem., submitted.

[58] K. Jinno, J. Wu, H. Sawada, Y. Kiso, J. High Resol. Chromatogr. 1998, $21,617$. 